\title{
openheart On site assessment of cardiac function and neural regulation in amateur half marathon runners
}

\author{
Laura Dalla Vecchia, ${ }^{1}$ Egidio Traversi, ${ }^{2}$ Alberto Porta, ${ }^{3}$ Daniela Lucini, ${ }^{4,5}$ \\ Massimo Pagani ${ }^{5}$
}

To cite: Dalla Vecchia $L$, Traversi E, Porta A, et al. On site assessment of cardiac function and neural regulation in amateur half marathon runners. Open Heart 2014;1:e00005. doi:10.1136/openhrt-2013000005

Received 20 November 2013 Revised 17 December 2013 Accepted 18 December 2013

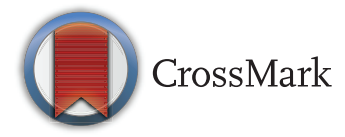

For numbered affiliations see end of article.

Correspondence to Dr Laura Dalla Vecchia; laura.dallavecchia@fsm.it

\section{ABSTRACT}

Objective: Strenuous exercise variably modifies cardiovascular function. Only few data are available on intermediate levels of effort. We therefore planned a study in order to address the hypothesis that a half marathon distance would result in transient changes of cardiac mechanics, neural regulation and biochemical profile suggestive of a complex, integrated adaptation.

Methods: We enrolled 35 amateur athletes (42 \pm 7 years). Supine and standing heart rate variability and a complete echocardiographic evaluation were assessed on site after the completion of a half marathon (postrace) and about 1 month after (baseline). Biochemical tests were also measured postrace.

Results: Compared to baseline, the postrace left ventricular end-diastolic volume was smaller, peak velocity of $E$ wave was lower, peak velocity of $A$ wave higher, and accordingly the E/A ratio lower. The postrace heart and respiratory rate were higher and variance of RR interval lower, together with a clear shift towards a sympathetic predominance in supine position and a preserved response to orthostasis. At baseline, athletes were characterised by a lower, although still predominant, sympathetic drive with a preserved physiological response to standing.

Conclusions: Immediately after a half marathon there are clear marks that an elevated sympathetic cardiac drive outlasts the performance, together with decreased left ventricular diastolic volumes and slight modifications of the left ventricular filling pattern without additional signs of diastolic dysfunction or indices of transient left or right ventricular systolic abnormalities. Furthermore, no biochemical indices of any permanent cardiac damage were found.

\section{INTRODUCTION}

The understanding and insight of exerciseinduced cardiovascular modifications is nowadays of great interest. It is known that exercise variably modifies cardiac function and cardiovascular neural regulation. These modifications can widely vary from favourable and protective to dangerous and hazardous. ${ }^{1}$ Such a broad range of effects may depend on individual risk profile on one hand and level

\section{KEY MESSAGES}

- The understanding and insight of exerciseinduced cardiovascular modifications is nowadays of great interest. It is known that marathon can determine transient elevations in cardiac biomarkers and echocardiographic evidence of different degrees of cardiac dysfunction. We therefore planned a study to assess cardiac function and neural regulation on adult amateur athletes running a shorter distance.

- Immediately after a half marathon, we found signs of an elevated sympathetic cardiac drive, together with decreased left ventricular diastolic volumes and slight modifications of the left ventricular filling pattern without additional signs of diastolic dysfunction, nor of transient left or right ventricular systolic abnormalities, nor biochemical indices of any permanent cardiac.

- Our study implies that a half marathon, although representing a much less stressful challenge and not producing major cardiac changes, determines slight diastolic modifications, whose relevance needs to be investigated. There is also evidence that a combined evaluation of cardiac mechanics and neural control may provide more complete information in guiding individual training and targets.

and type of exercise on the other hand. In the past recent years, studies carried on amateur athletes have fathomed the effects of strenuous exercise on cardiovascular function. $^{2-7}$ It has been demonstrated that elevations of cardiac biomarkers after strenuous endurance exercises can occur in the absence of sustained myocardial dysfunction. Conversely, signs of transient left ventricular (LV) diastolic impairment and/or right ventricular (RV) dysfunction have been detected in some cases. These reversible and functional changes have been attributed to the combination of prolonged myocardial workload, tachycardia and dehydration secondary to strenuous exercise. 
Most investigations are limited to the evaluation of cardiovascular changes occurring after marathon or ultramarathon races that represent an unusually elevated load to the heart. Only few data are available on relatively shorter distances, such as those run in a half marathon. ${ }^{8}$ Although still challenging, it is less demanding, and may represent a reasonable intermediate level of effort, which is increasingly followed by growing numbers of participants, eventually preceding a full marathon engagement. In addition, to our knowledge there are no studies which combine on site evaluation of mechanical and neural response to endurance races. Accordingly, we planned a study on a group of trained adult amateur athletes in order to address specifically the hypothesis that a half marathon distance would result in transient postrace changes of cardiac mechanics, neural regulation and biochemical profile suggestive of a complex, integrated adaptation.

\section{METHODS}

\section{Patient population}

We enrolled 35 amateur athletes, 4 women and 31 men, age $42 \pm 7$ years (range 30-57), body mass index 22.1 $\pm 1.6 \mathrm{~kg} / \mathrm{m}^{2}$, participating in the fifth Corripavia half marathon (distance $21097 \mathrm{~km}$ ), who volunteered and provided written, informed consent.

All athletes were healthy and free from pulmonary and cardiovascular disease and any family history of early cardiovascular disease and were not taking any prescribed medication.

\section{Protocol}

All participants were studied at the end of the half marathon completion (postrace) to measure onsite parameters and about 4 weeks after (baseline) to assess baseline parameters.

Postrace, according to the arrival order, runners were directed to one of the two stations prepared in front of the finishing line, where echocardiographic evaluation, supine and standing heart rate variability (HRV) assessment and blood sample withdrawal were performed. Supine brachial blood pressure was measured with a manual sphygmomanometer. HRV and echocardiography were repeated to assess baseline parameters a month after the race. As only 4 of 35 participants in the study agreed to repeat blood sample, this was avoided in all.

There was no fluid restriction during the race. In the period between the postrace and the baseline tests, runners were allowed to continue their regular training but were asked to avoid any race. The day of the baseline tests, each participant was interviewed to verify adherence to the training schedule (no race allowed) and the absence of intercurrent disease. On both days all participants had a caffeine-free breakfast, care was taken to avoid noise and the ambient temperature was kept comfortable during the tests.

\section{Echocardiographic assessment}

A comprehensive echocardiographic examination (twodimensional (2D), M-mode, Doppler, Tissue Doppler Imaging (TDI) and 2D Longitudinal Strain (LS)) was performed in accordance with recommendations from the American Society of Echocardiography by two experienced and qualified sonographers using a commercially available ultrasound system (Vivid 7, GE Medical, Horton, Norway) and a $1.5-4 \mathrm{MHz}$ phased array transducer. ${ }^{9}$ The images were digitally stored and later analysed offline using an EchoPAC V.7.0.0 (GE Vingmed). The presence of significant valvular disease or intracardiac shunts was excluded by means of colour Doppler. LV end-diastolic volume (EDV) and end-systolic volume (ESV), as well as biplane LV ejection fraction (EF), were calculated from four-chamber and twochamber images, using the modified Simpson rule. Mitral inflow measurements were obtained from the four-chamber view by placing the sample volume at mitral leaflet tips, and early filling $\mathrm{LV}$ velocity (E) and atrial filling velocity (A) waves, E/A ratio and $\mathrm{E}$ wave deceleration time (DT) were measured. TDI measurements were assessed in the apical four-chamber view placing the sample volume at the junction of the interventricular septum with the mitral anulus. Early $\left(\mathrm{e}^{\prime}\right)$ diastolic myocardial velocities were recorded and $\mathrm{E} / \mathrm{e}^{\prime}$ ratio was calculated. For 2D, TDI and 2DLS measurements three beats were stored and analysed. Segmental evaluation of peak systolic 2DLS was performed at the basal and middle levels for all the walls visualised in the fourchamber, three-chamber and two-chamber apical views. Values of all segments were averaged to obtain the global LS. ${ }^{10}$ All echocardiographic analyses were performed separately and blinded to other participant data by an experienced echocardiographer.

\section{HRV assessment}

ECG was obtained by a modified II lead configuration. Respiratory activity was recorded by a thoracic belt connected to a pressure transducer. ECG and respiratory activity signals were digitised at $300 \mathrm{~Hz}$ by an analog-to-digital board (AT-MIO 16E2, National Instruments) and stored on the hard disk of a personal computer for offline analysis. Signals were continuously recorded with the participant lying supine for $5 \mathrm{~min}$ and while standing for $4 \mathrm{~min}$.

Analog data were analysed offline. RR interval (RR) was calculated as the time distance between two consecutive interpolated parabolic $\mathbf{R}$ wave apexes. Software techniques for series extraction, spectral and cross-spectral analyses of RR and respiratory activity have been described in detail elsewhere. ${ }^{11}{ }^{12}$ Autoregressive spectral analysis provided the power spectrum density of the low frequency (LF, from 0.03 to $0.15 \mathrm{~Hz}$ ) and high frequency (HF, above $0.15 \mathrm{~Hz}$ ) components of the RR series. These oscillatory components were expressed in both absolute $\left(\mathrm{ms}^{2}\right.$ or $\left.\mathrm{mm} \mathrm{Hg}^{2}\right)$ and normalised units (nu). ${ }^{13}$ Normalisation was obtained dividing the 
absolute power of each component by total variance (minus the power of the very low frequency component) and then multiplying by $100 .{ }^{11-14}$ Spectral indices were indicated as $\mathrm{LF}_{\mathrm{RR}}, \mathrm{HF}_{\mathrm{RR}}$ or $\mathrm{LF} / \mathrm{HF}$. $\mathrm{LF}_{\mathrm{RR}}$ expressed in nu was utilised as an index of the cardiac sympathetic oscillatory modulation, $\mathrm{HF}_{\mathrm{RR}}$ as a marker of the vagal cardiac oscillatory modulation. ${ }^{12-14}$ The $\mathrm{LF}_{\mathrm{RR}} / \mathrm{HF}_{\mathrm{RR}}$ ratio quantified the sympathovagal interaction to the sinoatrial node. ${ }^{11-14}$

\section{Biochemical studies}

Blood samples were collected in K2EDTA tubes for blood count and in no additive tubes for serum cardiac biomarkers, stored in refrigerated transport box $\left(2-8^{\circ} \mathrm{C}\right)$ and arrived at the biochemistry laboratory in about $20 \mathrm{~min}$. On arrival whole blood was analysed for haematological parameters, and serum prepared by centrifugation and analysed thereafter. Creatine kinase (CK) was determined using an automated analyser (Roche Modular), and the Roche CK kit (Roche Diagnostics $\mathrm{GmbH}$, Mannheim, Germany). CK-MB isoenzyme and troponin I (cTnI) were performed using a one-step immunoassay sandwich method with a final fluorescent method (ELFA) on a Mini VIDAS (bioMérieux, Marcy-l'Etoile, France). The CK assay normal range was 38-174 U/L for men and 26-140 U/L for women, and the CK-MB assay detection limit was $6.8 \mathrm{ng} / \mathrm{mL}$. The cTnI assay detection limit was $0.01 \mu \mathrm{g} / \mathrm{L}$, with the clinical cut-off level for myocardial infarction set at $0.03 \mu \mathrm{g} / \mathrm{L}^{15}$

\section{Statistical analysis}

All the participants who volunteered were enrolled and analysed. Data are expressed as mean \pm SD. The significance of the differences between echocardiographic parameters (see list in table 1) was assesses via paired two-sided $\mathrm{t}$ test. If the normality test was not fulfilled according to a Kolmogorov-Smirnov test with $\mathrm{p}<0.05$, Wilcoxon-signed rank test was applied. The significance of the differences between HRV parameters at baseline and postrace, measured in supine position and while standing (see list in table 2) was evaluated using two-way repeated measures analysis of variance with the Holm-Sidak post hoc test. We assessed the effect of race within the same experimental condition (ie, supine or standing) and the effect of the modification of posture within the same period of analysis (ie, postrace or baseline examination). The appearance of cTnI in the systemic circulation above assay detection levels was analysed descriptively as it was reported only in one case. A p $<0.05$ was considered significant. All analyses were carried out on SPSS (Statistical Package for Social Sciences, Chicago, Illinois, USA), V.17.0.

\section{RESULTS}

All 35 enrolled runners finished the race, were ambulatory, and reported no signs or symptoms of (pre)
Table 1 Descriptive statistics of echocardiographic variables of amateur athletes assessed 1 month after the race (baseline) and on site after the half marathon completion (postrace)

\begin{tabular}{|c|c|c|}
\hline & Baseline & Postrace \\
\hline HR (bpm) & $63 \pm 12$ & $86 \pm 14^{*}$ \\
\hline $\mathrm{SAP}(\mathrm{mm} \mathrm{Hg})$ & $121 \pm 8$ & $108 \pm 8^{*}$ \\
\hline LV EDV $\left(\mathrm{cm}^{3}\right)$ & $117 \pm 24$ & $105 \pm 23^{*}$ \\
\hline LV ESV $\left(\mathrm{cm}^{3}\right)$ & $43 \pm 11$ & $41 \pm 14$ \\
\hline LV EF (\%) & $62 \pm 6$ & $62 \pm 7$ \\
\hline LV mass index $\left(\mathrm{g} / \mathrm{m}^{2}\right)$ & $177 \pm 46$ & $164 \pm 41$ \\
\hline GLS (\%) & $-20 \pm 3$ & $-19 \pm 3$ \\
\hline Aortic root diameter (mm) & $32 \pm 3$ & $26 \pm 3$ \\
\hline Max LA area $\left(\mathrm{cm}^{2}\right)$ & $15 \pm 3$ & $14 \pm 2$ \\
\hline Min LA area $\left(\mathrm{cm}^{2}\right)$ & $8 \pm 2$ & $8 \pm 2$ \\
\hline RVOT prox (mm) & $30 \pm 3$ & $29 \pm 3$ \\
\hline RVI diameter (mm) & $33 \pm 2$ & $32 \pm 3$ \\
\hline TAPSE $(\mathrm{mm})$ & $23 \pm 1$ & $24 \pm 1$ \\
\hline$E(m / s)$ & $0.74 \pm 0.13$ & $0.67 \pm 0.15^{\star}$ \\
\hline$A(m / s)$ & $0.52 \pm 0.13$ & $0.63 \pm 0.15^{\star}$ \\
\hline E/A ratio & $1.5 \pm 0.36$ & $1.08 \pm 0.18^{*}$ \\
\hline DT (ms) & $211.22 \pm 38.47$ & $198.46 \pm 41.8$ \\
\hline $\mathrm{E}^{\prime}(\mathrm{cm} / \mathrm{s})$ & $10.07 \pm 1.4$ & $9.29 \pm 2.1$ \\
\hline E/e' ratio & $7.39 \pm 1.09$ & $7.41 \pm 1.8$ \\
\hline ICV diameter (mm) & $20 \pm 2$ & $20 \pm 3$ \\
\hline
\end{tabular}

Data represent mean $\pm S D,{ }^{*} p<0.05$ postrace versus baseline. A, mitral A wave filling velocity; DT, mitral deceleration time; $e^{\prime}$, early diastolic myocardial velocity; $E$, mitral $E$ wave filling velocity; EDV, end-diastolic volume; EF, ejection fraction; ESV, end-systolic volume; GLS, global longitudinal strain; HR, heart rate; ICV, inferior cava vein; LA, left atrium; LV, left ventricular; RVI, right ventricular inflow; RVOT prox, right ventricular outflow tract proximal diameter; SAP, systolic arterial pressure; TAPSE, tricuspid anular plane systolic excursion.

syncope or cardiovascular impairment. None required medical attention.

Race started at 9:00, the average finish time was $96 \mathrm{~min}$ and $30 \mathrm{~s}$ (range $78^{\prime} 55^{\prime \prime}$ to $119^{\prime} 42^{\prime \prime}$ ). Weather conditions were mild with mean temperature of $15^{\circ} \mathrm{C}$.

The time from cessation of running to onsite HRV and echocardiographic assessments was similar among all runners $(15-20 \mathrm{~min})$. The time from the race day to the baseline tests was $30 \pm 8$ days.

Compared to baseline, as expected, postrace supine HR was higher by $22 \pm 14 \mathrm{bpm}(\mathrm{p}<0.05)$ and supine systolic arterial pressure lower by $13 \pm 8 \mathrm{~mm} \mathrm{Hg} \quad(p<0.05$; table 1).

\section{Postrace and baseline echocardiographic parameters}

Echocardiographic parameters are shown in table 1 . Compared to baseline, postrace LVEDV was smaller by $11.63 \pm 3.82 \mathrm{~mL}(\mathrm{p}<0.05)$, while LVESV, LV mass, as well as the indices of LV systolic function, including assessment of regional wall motion by TDI and 2DLS (figure 1) were similar. Aortic root diameter, left atrial areas, RV dimension and systolic function were also unchanged. Peak velocity of $\mathrm{E}$ wave was found lower by $0.7 \pm 4.6 \mathrm{~m} / \mathrm{s}$, peak velocity of A wave was found higher by 
Table 2 Descriptive statistics of heart rate variability indices assessed 1 month after the race (baseline) and on site after the half marathon completion (postrace)

\begin{tabular}{|c|c|c|c|c|}
\hline & \multicolumn{2}{|l|}{ Baseline } & \multicolumn{2}{|l|}{ Postrace } \\
\hline & Supine & Standing & Supine & Standing \\
\hline HR (bpm) & $63 \pm 12$ & $74 \pm 16^{\star \star}$ & $86 \pm 14^{*}$ & $97 \pm 16^{\star}, * \star$ \\
\hline Resp (cycles/min) & $16 \pm 3$ & $15 \pm 1$ & $18 \pm 1^{*}$ & $19 \pm 1^{*}$ \\
\hline $\mathrm{RR}(\mathrm{ms})$ & $993 \pm 183$ & $842 \pm 168^{\star \star}$ & $730 \pm 127^{*}$ & $643 \pm 110^{*}$, ** \\
\hline$\sigma_{\mathrm{RR}}^{2}\left(\mathrm{~ms}^{2}\right)$ & $3874 \pm 788$ & $3044 \pm 852$ & $1567 \pm 586^{*}$ & $1671 \pm 823^{*}$ \\
\hline $\mathrm{LF}_{\mathrm{RR}}\left(\mathrm{ms}^{2}\right)$ & $1026 \pm 113$ & $1455 \pm 319$ & $1001 \pm 448$ & $1431 \pm 148$ \\
\hline $\mathrm{LF}_{\mathrm{RR}}(\mathrm{nu})$ & $59 \pm 12$ & $81 \pm 16^{\star \star}$ & $76 \pm 13^{*}$ & $86 \pm 10^{\star *}$ \\
\hline $\mathrm{HF}_{\mathrm{RR}}\left(\mathrm{ms}^{2}\right)$ & $646 \pm 130$ & $357 \pm 45$ & $132 \pm 97^{*}$ & $178 \pm 88$ \\
\hline $\mathrm{HF}_{\mathrm{RR}}(\mathrm{nu})$ & $37 \pm 22$ & $17 \pm 6^{\star \star}$ & $14 \pm 9^{*}$ & $8 \pm 7^{*}, * *$ \\
\hline LF/HR ratio & $3 \pm 3$ & $15 \pm 4^{\star *}$ & $11 \pm 2^{*}$ & $34 \pm 4^{\star *}$ \\
\hline
\end{tabular}

Data represent mean $\pm S D$, ${ }^{*} p<0.05$ postrace versus baseline; ${ }^{* *} p<0.05$ standing versus supine.

$\sigma_{R R}^{2}$, variance of RR variability; $H_{R R}$, high-frequency power of RR variability; HR, heart rate; LF $F_{R R}$, low-frequency power of RR variability; nu, normalised units; RR, R-R interval; Resp, respiratory activity.

$15.6 \pm 3.5 \mathrm{~m} / \mathrm{s}$, and accordingly the $\mathrm{E} / \mathrm{A}$ ratio lower by $0.29 \pm 0.09(\mathrm{p}<0.05)$, while $\mathrm{DT}, \mathrm{e}^{\prime}$ and $\mathrm{E} / \mathrm{e}^{\prime}$ ratio were analogous. The inferior cava vein (ICV) diameter was similar in both conditions with a preserved $>50 \%$ inspiratory collapse reflecting normal central venous pressure.

\section{Postrace and baseline HRV}

HRV data are shown in table 2. At baseline, athletes demonstrated signs of sympathetic predominance when supine with a preserved excitatory physiological response to standing, as $\mathrm{HR}$ increased by $11 \pm 1 \mathrm{bpm}, \mathrm{LF}_{\mathrm{RR}}$ increased by $21 \pm 3 \mathrm{nu}$ and $\mathrm{HF}_{\mathrm{RR}}$ decreased by $19 \pm 3 \mathrm{nu}$, and accordingly LF/HF ratio increased by $12 \pm 4(\mathrm{p}<0.05$; table 2, figure 2). Compared to baseline, as expected, postrace $\mathrm{HR}$ and respiratory rate were higher $(\mathrm{p}<0.05)$, while variance of RR was lower $(p<0.05)$. Postrace autonomic profile was also characterised by other significant differences compared to baseline. Indeed, we observed a clear shift towards higher indirect indices of prevailing sympathetic modulation in supine position $\left(\mathrm{LF}_{\mathrm{RR}}\right.$ higher by $19 \pm 4 \mathrm{nu}$ and $\mathrm{HF}_{\mathrm{RR}}$ lower by $31 \pm 5 \mathrm{nu}$, postrace vs baseline; $\mathrm{p}<0.05)$ despite an HR in normal range. The postrace response to orthostasis was still clear, as HR increased by $10 \pm 1 \mathrm{bpm}, \mathrm{LF}_{\mathrm{RR}}$ increased by $10 \pm 2 \mathrm{nu}$ and $\mathrm{HF}_{\mathrm{RR}}$ decreased by $5 \pm 2 \mathrm{nu}$, and accordingly $\mathrm{LF} / \mathrm{HF}$ ratio increased by $11 \pm 4(\mathrm{p}<0.05$; table 2 , figure 2$)$.

\section{Postrace biochemical parameters}

Postrace, mean blood levels for haemoglobin and haematocrit resulted within normal limits $(14.8 \pm 1.1 \mathrm{~g} / \mathrm{dL}$ and $43.3 \pm 2.9 \%$, respectively). Mean white cell count was 14.14 $\pm 3.9 \times 10^{\wedge} 9 / \mathrm{L}$ with a range from normal to abnormally high values (from 8.9 to $23.3 \times 10^{\wedge} 9 / \mathrm{L}$ ). Value above normal limits were present in 26 athletes (74\%), while the remaining showed normal values $\left(15.5 \pm 3.4 \times 10^{\wedge} 9 / \mathrm{L}\right.$, range $10.6-23.3 \times 10^{\wedge} 9 / \mathrm{L}$ and $9.4 \pm 0.4 \times 10^{\wedge} 9 / \mathrm{L}$, range 8.9 $10.2 \times 10^{\wedge} 9 / \mathrm{L}$, respectively). Postrace, CK was above normal limits in all participants with a mean value of $320.3 \pm 176.3 \mathrm{U} / \mathrm{L}$, range $121-913 \mathrm{U} / \mathrm{L}$, while $\mathrm{CK}-\mathrm{MB}$ was above normal limits only in 16 of 35 participants. In this latter group mean CK-MB was $9.6 \pm 2 \mathrm{ng} / \mathrm{mL}$ (range 6.9$15.9 \mathrm{ng} / \mathrm{dL})$, while in the remaining 19 participants it was $4.2 \pm 1.3 \mathrm{ng} / \mathrm{dL}$ (range $1.8-6.3 \mathrm{ng} / \mathrm{dL}$ ). Detectable
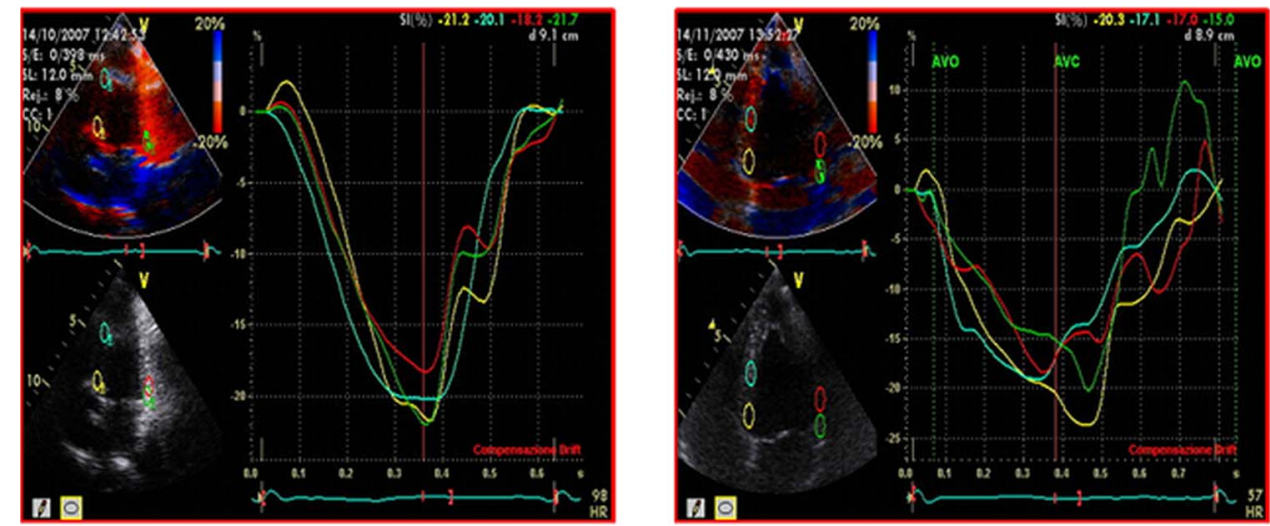

Figure 1 An exemplar scan from tissue-Doppler assessment of regional wall strain in the apical four-chamber view measured at the basal and at the mid-wall level of the septum and lateral wall obtained in an amateur athlete immediately after the completion of the half marathon (postrace, left panel) and 1 month after (baseline, right panel). 

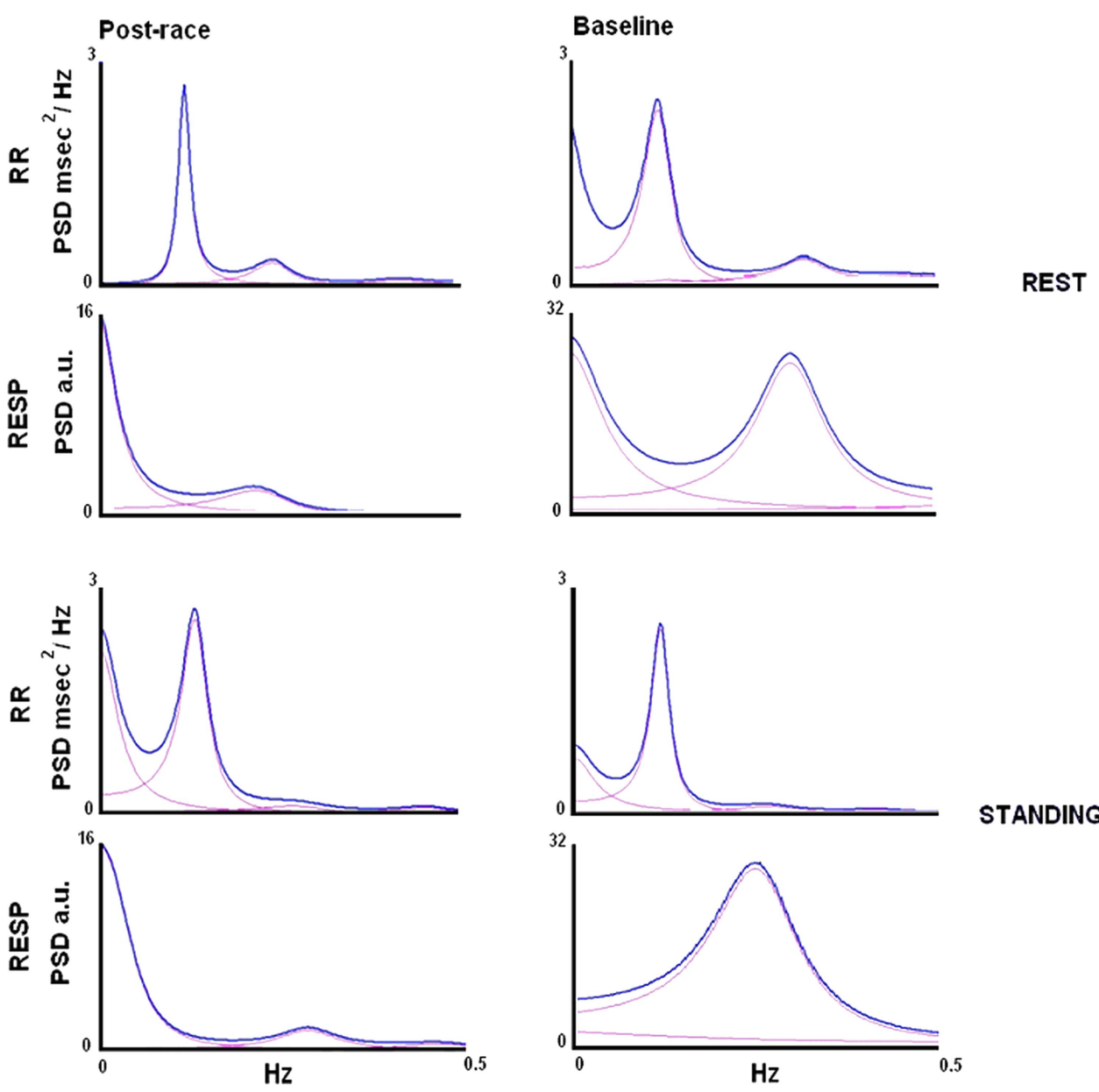

Figure 2 Examples of power spectrum analyses of RR and RESP in an amateur athlete (same participant as in figure 1) few minutes after the completion of the half marathon (postrace, left panels) and 1 month after (baseline, right panels) at rest (top panels) and during standing (bottom panels). Baseline autonomic profile is characterised by sympathetic predominance at rest both postrace (left top panel) and at baseline (right top panel). In both conditions the physiological excitatory response to orthostasis is preserved (bottom panels). a.u., arbitrary units; PSD, power spectrum density; RESP, respiratory activity; RR, R-R interval.

cTnI was observed only in one 32-year-old male athlete $(0.45 \mu \mathrm{g} / \mathrm{L})$, who did not show any difference for the echocardiographic and HRV parameters compared to the other runners either postrace or at baseline.

\section{DISCUSSION}

The major finding of this study was that immediately after a half marathon there are clear signs that an elevated sympathetic cardiac drive outlasts the performance, together with decreased LV diastolic volumes and slight modifications of the LV filling pattern without additional signs of diastolic dysfunction. Neither echocardiographic nor biochemical indices of any permanent cardiac damage, nor indices of transient or RV systolic abnormalities were found.

\section{Postrace echocardiographic findings}

Previous studies have demonstrated that after a marathon challenge transient cardiac diastolic dysfunction can occur in some cases, possibly due to an excessive load to the heart. ${ }^{2-7}$ In addition, the more intense the exercise load, the higher the cardiac mechanical susceptibility and more persistent the diastolic dysfunction of both the LV and the RV. ${ }^{2}{ }^{4}{ }^{16}$ In our study after a half marathon, which represents a much less stressful challenge, we detected a decreased LV diastolic volume and a decrease in the $\mathrm{E} / \mathrm{A}$ ratio, although still within the normal ranges $(>1)$, due to a decrease of the $\mathrm{E}$ wave and an increase of the A wave. Other and more sophisticated measures of LV diastolic properties, such as DT, $\mathrm{E}^{\prime}$ wave, $\mathrm{E} / \mathrm{e}^{\prime}$ ratio, LA size and reservoir, were not different from values observed at baseline. The $\mathrm{E}$ wave is known to be preload dependent, and the A wave dependent on heart rates and volume loads. ${ }^{17-21}$ Indeed, the A wave amplitude may be elevated during higher heart rates because of the merging of the $\mathrm{E}$ and $\mathrm{A}$ wave ${ }^{171821}$ and because of the presence of smaller LVEDV. ${ }^{17} 2021$ This latter finding can be consequent to a reduced preload resulting from postexercise redistribution of blood to the 
periphery. ${ }^{21}$ Reduced preload might also depend on dehydration-induced decrements in central blood volume, although in our middle-distance marathoners there were no signs of significantly reduced central venous pressure as assessed by ICV diameter and collapsibility. The impact of acute exercise on diastolic function could also be viewed as a process of augmentation to underpin increases in LV systolic function ${ }^{18}{ }^{19}$ or decreases in RV systolic function. ${ }^{8}$ Therefore, the isolated modification of the transmitral inflow pattern might reflect an adaptation to the postexercise haemodynamic changes rather than intrinsic alterations in $\mathrm{LV}$ relaxation or compliance properties. ${ }^{17-20}$ On the other hand, diastolic modifications might reflect reduced lusitropic properties for instance as a residual postexercise altered sodium-calcium handling. ${ }^{22}$ Despite speculation at the moment there is no evidence to support any of these competing theories. This requires further study in a range of different populations.

In keeping with previous investigations, ${ }^{2-7}$ the echocardiographic indices of regional and global systolic function were normal at baseline and unchanged postrace. Importantly, strain analysis provided an objective measure of preserved segmental LV systolic shortening. As previously demonstrated these indices have excellent feasibility and reproducibility, ${ }^{10}{ }^{23}$ are considered superior to LVEF and visual wall motion assessment for prediction of mortality, ${ }^{24}$ and have been recently demonstrated as accurate indicators for patients with a potential for substantial myocardial damage. ${ }^{25}$

We therefore demonstrated that after a half marathon not only the LV systolic function is preserved, but also that only minor signs of diastolic impairment are detectable. Indeed, the reduced load imposed by the half marathon distance as compared to a full marathon, seems to preserve the heart from excessive stress and major diastolic impairment.

\section{Postrace autonomic cardiac control}

Spectral analysis of short-term HRV has been shown to be capable of detecting the complex adaptive changes in sympathovagal balance attending various aspects of exercise and recovery in athletes. ${ }^{26-28}$ In the volunteers of the present study we found at baseline a supine spectral profile characterised by a slight predominance of $\mathrm{LF}_{\mathrm{RR}}$ expressed in $\mathrm{nu}$, in the presence of heart rates in the low-normal range $(63 \pm 12 \mathrm{bpm})$, as observed in welltrained, near competition athletes. ${ }^{27}$ Postrace, immediately after the completion of the half marathon, that is, after a heavy bout of acute exercise, an even more pronounced predominance of indirect indices of sympathetic modulation to the heart was present in the supine position despite, again as expected in athletes, the heart rate, although higher than at baseline, was still in the normal range $(86 \pm 14 \mathrm{bpm})$. Previous studies have demonstrated that training leads to an array of possible sympathovagal adjustments, that is, regular training at intermediate load leads to training bradycardia and vagal predominance, ${ }^{29}$ while a strenuous training for getting ready to competition enhances sympathetic modulation. ${ }^{26}{ }^{27}$ Signs of elevated sympathetic modulation have even been correlated to faster marathon arrival time. ${ }^{3031}$

It is noteworthy that, either at baseline or postrace, our athletes showed a physiological and adequate response to orthostasis, further supporting the notion of a physiological rather than pathological response in our population.

\section{Biomarkers}

As baseline biochemistry was not assessed we may only suggest some comments regarding postrace biochemical findings.

High levels of leucocytes were detected in $74 \%$ of our non-elite runners after the half marathon completion. It has been previously reported that acute strenuous exercise can be associated with transient leucocytosis, followed by complete normalisation after $24 \mathrm{~h}$, although its significance has not been completely understood. ${ }^{32}$

Conversely, levels of haemoglobin and haematocrit were found within normal ranges. These findings, together with normal ICV diameters and collapsibility, suggest that, on the whole, significant dehydration did not develop during the race.

CK was elevated in all participants. Although CK is not cardiac specific, it parallels the strenuous nature of a half marathon. Regarding markers of myocardial injury, CK-MB was high in $46 \%$ of our athletes, while all, but one, were characterised by normal levels of cTnI after the race, further supporting the interpretation that the half marathon may represent a limited load. Indeed, previous investigations have reported increased levels of CK-MB and troponins in elite and non-elite athletes following the completion of marathon events, ${ }^{2}{ }^{33-35}$ that is, a double distance than the one we tested in the present study, and correlated with the increased endurance time. ${ }^{2}$ This hypothesis is in accordance with our results, as we can speculate that a longer distance might increase the number of participants with positive biomarkers. Anyhow, it has been demonstrated that the postexercise troponin release does not correspond to true myocardial necrosis. ${ }^{358}{ }^{34}$ It may represent reversible cardiomyocyte membrane damage due to an excessive cardiac load, as it is not associated with any sustained alterations of echocardiographic parameters of systolic, diastolic or right-sided heart dysfunction, as it was the case in our athletes. Notably, the racer in our study with slight elevation of cTnI was healthy, well-trained and still running competitions at a 2-year follow-up.

\section{Limitations}

Our study has several limitations mainly due to the impossibility to standardise experimental conditions, such as fluid intake during the race or level of training for a baseline evaluation. In addition, we limited the number of echocardiographic and HRV measures in 
order to allow a similar and as fast as possible postrace on site evaluation. For example, HRV was not integrated by the beat-to-beat blood pressure variability assessment and echocardiographic RV assessment was partial. Finally, the lack of baseline biomarkers limits the interpretation of such variables. However, combining the evaluation of cardiac mechanics and neural control may represent a key to a better understanding of those important adaptational processes that are engaged to different levels of exercise challenge.

\section{Implications for clinical practice}

While regular physical activity is clearly a positive determinant of longevity in recreational athletes and former endurance athletes and is associated with an improved cardiovascular risk profile, ${ }^{1}$ debate exists on the level of exercise to be recommended at different ages and in the presence of cardiovascular risk and/or diagnosed cardiovascular disease. ${ }^{136} 37$ The intensity of exercise is also a key issue in cardiac rehabilitation. ${ }^{38}$

Indeed exercise in general can be considered a therapy and its beneficial effects overcome potential risks when correctly prescribed.

Former studies have demonstrated that marathon and ultramarathon can determine elevations in cardiac biomarkers and echocardiographic evidence of different degrees of cardiac dysfunction, and thus they may represent a potentially dangerous load $^{2-7}$ However, it has been suggested that appropriate training and preparation play an important protective role against excessive endurance exercise loads. ${ }^{2} 17$

Our study implies that a half marathon, although representing a much less stressful challenge and not producing major cardiac changes, still determines slight diastolic modifications, whose relevance needs to be investigated. There is also evidence that a combined evaluation of cardiac mechanics and neural control may provide more complete information in guiding individual training and targets.

\section{Author affiliations \\ ${ }^{1}$ IRCCS Fondazione Salvatore Maugeri, Istituto Scientifico di Milano, Milan, Italy \\ ${ }^{2}$ IRCCS Fondazione Salvatore Maugeri, Istituto Scientifico di Montescano, Pavia, Italy \\ ${ }^{3}$ Department of Biomedical Sciences for Health, Galeazzi Orthopedic Institute, University of Milan, Milan, Italy \\ ${ }^{4}$ IRCCS Istituto Clinico Humanitas, Sezione Medicina dell'Esercizio e Sindromi Funzionali, Rozzano, Milan, Italy \\ ${ }^{5}$ Centro di ricerca Terapia Neurovegetativa e Medicina dell'esercizio, University of Milan, U.O. Telemedicina e Medicina dello Sport, Ospedale 'Luigi Sacco', Milano, Italy}

Acknowledgements The authors would like to thank all the runners who have participated in the study. They thank Franco Corona, President of the Asd Atletica Cento Torri Pavia for the organisation and technical support. The authors are grateful to Miriam De Giorgi and Giorgio Zanoni for the echocardiographic acquisition and technical support and to Gabriella Martino for the support in laboratory measurements.
Contributors All authors have taken due care to ensure the integrity of the work, meet authorship criteria of the Uniform Requirements, as follows: LDV and ET participated in conception and design of the study; LDV, ET and DL participated in clinical assessment and follow-up; LDV, AP and MP participated in analysis and interpretation of the data; LDV, ET, DL, AP and MP participated in drafting of the manuscript. All authors have seen and approved the manuscript.

Funding This research received no specific grant from any funding agency in the public, commercial or not-for-profit sectors.

\section{Competing interests None.}

Ethics approval The study conformed to the standards set by the declaration of Helsinki and ethical approval was obtained from the local Ethics Committee.

Provenance and peer review Not commissioned; externally peer reviewed.

Data sharing statement No additional data are available.

Open Access This is an Open Access article distributed in accordance with the Creative Commons Attribution Non Commercial (CC BY-NC 3.0) license, which permits others to distribute, remix, adapt, build upon this work noncommercially, and license their derivative works on different terms, provided the original work is properly cited and the use is non-commercial. See: http:// creativecommons.org/licenses/by-nc/3.0/

\section{REFERENCES}

1. Thompson PD, Franklin BA, Balady GJ, et al. American Heart Association Council on Nutrition, Physical Activity, and Metabolism; American Heart Association Council on Clinical Cardiology; American College of Sports Medicine. Exercise and acute cardiovascular events placing the risks into perspective: a scientific statement from the American Heart Association Council on Nutrition, Physical Activity, and Metabolism and the Council on Clinical Cardiology. Circulation 2007;115:2358-68.

2. Neilan TG, Januzzi JL, Lee-Lewandrowski E, et al. Myocardial injury and ventricular dysfunction related to training levels among nonelite participants in the Boston marathon. Circulation 2006;114:2325-33.

3. Dawson EA, Whyte GP, Black MA, et al. Changes in vascular and cardiac function after prolonged strenuous exercise in humans. J Appl Physiol 2008;105:1562-8.

4. Neilan TG, Yoerger DM, Douglas PS, et al. Persistent and reversible cardiac dysfunction among amateur marathon runners. Eur Heart $J$ 2006;27:1079-84.

5. Knebel F, Schimke I, Schroeckh S, et al. Myocardial function in olde male amateur marathon runners: assessment by tissue Doppler echocardiography, speckle tracking, and cardiac biomarkers. J Am Soc Echocardiogr 2009;22:803-9.

6. König D, Schumacher YO, Heinrich L, et al. Myocardial stress after competitive exercise in professional road cyclists. Med Sci Sports Exerc 2003;35:1679-83.

7. Scott JM, Esch BT, Shave R, et al. Cardiovascular consequences of completing a $160-\mathrm{km}$ ultramarathon. Med Sci Sports Exerc 2009;41:26-34.

8. Oomah SR, Mousavi N, Bhullar N, et al. The role of three-dimensional echocardiography in the assessment of right ventricular dysfunction after a half marathon: comparison with cardiac magnetic resonance imaging. J Am Soc Echocardiogr 2011;24:207-13.

9. Lang RM, Bierig M, Devereux RB, et al.; Chamber Quantification Writing Group; American Society of Echocardiography's Guidelines and Standards Committee; European Association of Echocardiography. Recommendations for chamber quantification: a report from the American Society of Echocardiography's Guidelines and Standards Committee and the Chamber Quantification Writing Group, developed in conjunction with the European Association of Echocardiography, a branch of the European Society of Cardiology. J Am Soc Echocardiogr 2005;18:1440-63.

10. EeK C, Grenne B, Brunvand $\mathrm{H}$, et al. Strain echocardiography predicts acute coronary occlusion in patients with non-ST-segment elevation acute coronary syndrome. Eur J Echocardiogr 2010;11:501-8.

11. Pagani M, Lombardi F, Guzzetti S, et al. Power spectral analysis of heart rate and arterial pressure variabilities as a marker of sympatho-vagal interaction in man and conscious dog. Circ Res 1986;59:178-93. 
12. Malliani A, Pagani M, Lombardi F, et al. Cardiovascular neural regulation explored in the frequency domain. Circulation 1991;84:482-92.

13. Task Force of the European Society of Cardiology and the North American Society of Pacing and Electrophysiology. Heart rate variability: standards of measurement, physiological interpretation and clinical use. Circulation 1996;93:1043-65

14. Lucini D, Mela GS, Malliani A, et al. Impairment in cardiac autonomic regulation preceding arterial hypertension in humans: insights from spectral analysis of beat-by-beat cardiovascular variability. Circulation 2002;106:2673-9.

15. Apple FS, Quist HE, Doyle PJ, et al. Plasma 99th percentile reference limits for cardiac troponin and creatine kinase MB mass for use with European Society of Cardiology/American College of Cardiology consensus recommendations. Clin Chem 2003;49:1331-6

16. Middleton N, Shave R, George K, et al. Left ventricular function immediately following prolonged exercise: a meta-analysis. Med Sci Sports Exerc 2006;38:681-7.

17. George KP, Naylor LH, Whyte GP, et al. Diastolic function in healthy humans: non-invasive assessment and the impact of acute and chronic exercise. Eur J Appl Physiol 2010;108:1-14.

18. George K, Oxborough D, Forster J, et al. Mitral annular myocardial velocity assessment of segmental left ventricular diastolic function after prolonged exercise in humans. J Physiol 2005;569(Pt 1): 305-13.

19. Scott JM, Warburton DE. Mechanisms underpinning exercise induced changes in left ventricular function. Med Sci Sports Exerc 2008;40:1400-7.

20. Burns AT, Connelly KA, La Gerche A, et al. Effect of heart rate on tissue Doppler measures of diastolic function. Echocardiography 2007;24:697-701.

21. Hart E, Shave R, Middleton N, et al. Effect of preload augmentation on pulsed wave and tissue Doppler echocardiographic indices of diastolic function after a marathon. J Am Soc Echocardiogr 2007;20:1393-9.

22. Mace LC, Palmer BM, Brown DA, et al. Influence of age and run training on cardiac $\mathrm{Na}+/ \mathrm{Ca} 2+$ exchange. J Appl Physiol 2003;95:1994-2003

23. Sjøli B, Ørn S, Grenne B, et al. Comparison of left ventricula ejection fraction and left ventricular global strain as determinants of infarct size in patients with acute myocardial infarction. J Am Soc Echocardiogr 2009;22:1232-8.

24. Stanton T, Leano R, Marwick TH. Prediction of all-cause mortality from global longitudinal speckle strain: comparison with ejection fraction and wall motion scoring. Circ Cardiovasc Imaging 2009:2:356-64.
25. Møller JE, Hillis GS, Oh JK, et al. Wall motion score index and ejection fraction for risk stratification after acute myocardial infarction. Am Heart J 2006;151:419-25.

26. Iellamo F, Legramante JM, Pigozzi F, et al. Conversion from vagal to sympathetic predominance with strenuous training in high-performance world class athletes. Circulation 2002;105:2719-24.

27. Furlan R, Piazza S, Dell'Orto S, et al. Early and late effects of exercise and athletic training on neural mechanisms controlling heart rate. Cardiovasc Res 1993;27:482-8.

28. Portier H, Louisy F, Laude D, et al. Intense endurance training on heart rate and blood pressure variability in runners. Med Sci Sports Exerc 2001;33:1120-5.

29. Sandercock GR, Bromley PD, Brodie DA. Effects of exercise on heart rate variability: inferences from meta-analysis. Med Sci Sports Exerc 2005;37:433-9.

30. Manzi V, Castagna C, Padua E, et al. Dose-response relationship of autonomic nervous system responses to individualized training impulse in marathon runners. Am J Physiol Heart Circ Physiol 2009;296:H1733-40.

31. Pagani M, Lucini D. Can autonomic monitoring predict results in distance runners? Am J Physiol Heart Circ Physiol 2009;296:H1721-2.

32. Lippi G, Banfi G, Montagnana M, et al. Acute variation of leucocytes counts following a half-marathon run. Int J Lab Hematol 2010;32(1 Pt 2):117-21.

33. Frassl W, Kowoll R, Katz N, et al. Cardiac markers (BNP, NT-pro-BNP, troponin I, troponin T, in female amateur runners before and up until three days after a marathon. Clin Lab 2008;54:81-7.

34. Middleton N, George K, Whyte G, et al. Cardiac troponin T release is stimulated by endurance exercise in healthy humans. J Am Coll Cardiol 2008;52:1813-14.

35. Jassal DS, Moffat D, Krahn J, et al. Cardiac injury markers in non-elite marathon runners. Int $J$ Sports Med 2009;30:75-9.

36. Hauser M, Petzuch K, Kühn A, et al. The Munich Triathlon Heart Study: ventricular function, myocardial velocities, and two-dimensional strain in healthy children before and after endurance stress. Pediatr Cardiol 2013;34:576-82.

37. Karlstedt E, Chelvanathan A, Da Silva M, et al. The impact of repeated marathon running on cardiovascular function in the aging population. J Cardiovasc Magn Reson 2012;14:58.

38. Mezzani A, Hamm LF, Jones AM, et al. Aerobic exercise intensity assessment and prescription in cardiac rehabilitation: a joint position statement of the European Association for Cardiovascular Prevention and Rehabilitation, the American Association of Cardiovascular and Pulmonary Rehabilitation and the Canadian Association of Cardiac Rehabilitation. Eur J Prev Cardiol 2013;20:442-67. 